\title{
Mecanismos de queda do fluxo em membranas de microfiltração de poliéterimida aplicadas à clarificação de água produzida sintética
}

\author{
Flux decline mechanisms in polyetherimide \\ microfiltration membranes for clarification \\ of synthetic produced water
}

Evelin Campos Verdolin Brandão ${ }^{1}$, Kátia Cecília de Souza Figueiredo ${ }^{1}$

\footnotetext{
${ }^{1}$ Departamento de Engenharia Química - EE/UFMG - CEP 31270-901, Belo Horizonte, MG, Brasil. e-mails: evelinverdolin@hotmail.com,katia@deq.ufmg.br
}

\section{RESUMO}

Membranas de microfiltração de poliéterimida foram preparadas pelo método de inversão de fases para controlar a espessura, porosidade e tamanho de poros dos filmes. 1-metil-2-pirrolidona foi utilizada como solvente e água como não solvente. As propriedades das membranas foram avaliadas por meio de microscopia eletrônica de varredura, microfiltração de água produzida sintética em modo frontal e tangencial, bem como mecanismos de bloqueio dos poros dos sistemas conforme descrito pelo modelo de Hermia. Os resultados mostraram que a morfologia das membranas foi significativamente alterada pela cinética de precipitação. $\mathrm{O}$ diâmetro médio de poros variou de 1,2 a 10,4 $\mu \mathrm{m}$, ambas com estrutura tipo dedos. Testes de permeação mostraram menor declínio do fluxo total para sistemas com escoamento tangencial $(89,5 \%)$ quando comparado com o escoamento frontal $(97,3 \%)$. Entretanto, o declínio reversível do fluxo foi maior do que o irreversível para ambos os sistemas. O coeficiente de rejeição foi maior do que 95,5\%, medido pela turbidez. Com relação aos mecanismos para o declínio do fluxo, observou-se que o bloqueio de poros intermediário foi capaz de explicar os resultados para a filtração frontal. O modelo de Hermia não se ajustou bem ao escoamento tangencial, provavelmente devido ao fato de o fluxo ter se estabilizado com o tempo. Os resultados mostraram o potencial do sistema para ser usado em microfiltração em longos prazos, desde que a lavagem periódica das membranas seja realizada.

Palavras-chave: escoamento frontal, escoamento tangencial, microfiltração, modelo de Hermia, polieterimida.

\begin{abstract}
Polyetherimide microfiltration membranes were prepared by phase inversion method aiming the control of film thickness, porosity and pore size. 1-methyl-2-pirrolidone was used as solvent and water as non-solvent. The properties of the membranes were addressed by means of scanning electron microscopy, dead-end and tangential flow microfiltration of a synthetic produced water effluent as well as the pore blocking mechanisms of the systems as described by Hermia models. The results showed that membrane morphology was significant modified by the kinetics of precipitation. The medium pore size ranged from 1.2 to $10.4 \mu \mathrm{m}$, both with finger-like structure. Permeation tests showed total flux decline lower for tangential flow mode $(89.5 \%)$ than the dead-end (97.3\%). However, the reversible flux decline was higher than the irreversible one in both systems. The rejection was higher than $95.5 \%$, which was addressed by turbidity. Regarding to the mechanisms to describe flux decline, it was evidenced that intermediate pore blocking was able to explain the results for dead-end filtration. Hermia model did not adjust to tangential flow mode, probably due to the stationary flux obtained with time. The results showed the potential of the system to be used in long term microfiltration, since the washing of the membranes is periodically observed.
\end{abstract}

Keywords: dead-end flow, Hermia model, microfiltration, polyetherimide, tangential flow. 


\section{INTRODUÇÃO}

Uma membrana pode ser definida como uma fina barreira capaz de controlar a taxa de permeação de espécies diferentes através de sua estrutura [1]. As membranas de microfiltração são utilizadas para a retenção de suspensões e emulsões, sendo que seus poros se encontram entre 0,1 e $10 \mu \mathrm{m}[2,3]$. A inversão de fases é o procedimento mais utilizado para a preparação das membranas poliméricas anisotrópicas [2,3]. Uma das formas mais comuns de induzir a separação de fases é pelo método de imersão/precipitação. De acordo com esta técnica, uma solução homogênea, composta por um polímero e seu solvente, é imersa em um banho de nãosolvente para o polímero, o que a tornará instável termodinamicamente. Assim, a solução será separada em duas fases, a fase sólida (rica em polímero) e a fase líquida (pobre em polímero). A primeira será responsável pela formação da matriz da membrana enquanto a fase líquida formará a estrutura porosa [1-4].

Membranas de poliéterimida, PEI, são de grande interesse para a separação de efluentes contendo contaminantes orgânicos, devido à elevada resistência química [5]. O preparo de membranas de PEI pela técnica de inversão de fases usando 1-metil-2-pirrolidona, NMP, como solvente e água como não solvente tem se mostrado uma alternativa bastante promissora visando modular as propriedades da barreira, tais como porosidade e estrutura de poros, de acordo com uma dada aplicação [6,7].

A microfiltração de água produzida de petróleo é um dos principais desafios para a tecnologia de membranas, considerando o volume do efluente gerado e as necessidades de operação por um longo período, sem queda significativa de fluxo permeado $[8,9]$.

Há dois modos de operação de um módulo de membranas: frontal ou tangencial [1-4]. Na filtração frontal, a alimentação é realizada perpendicular à superfície da membrana, de modo que as partículas em suspensão vão se acumulando nessa superfície. Este depósito gera a chamada torta de filtração [10]. No caso da filtração tangencial, a alimentação escoa paralelamente à superfície da membrana, enquanto o permeado passa transversalmente a ela [1-4]. Assim, apenas uma pequena parcela do fluido atravessa a membrana e sua velocidade de escoamento faz com o material retido na superfície seja removido por todo o tempo. Portanto, o acúmulo do material suspenso observado na filtração frontal é significativamente diminuído, evitando ou apenas retardando a formação da torta [1-4].

Durante o processo de separação através de membranas, dois fenômenos são cruciais para seu desempenho: a polarização de concentração e a incrustação (fouling). Estes fenômenos estão inter-relacionados e causam o aumento da resistência à transferência de massa na membrana e, como consequência, o declínio do fluxo de permeado [11]. A incrustação é a deposição irreversível de solutos dentro dos poros ou na sua superfície da membrana [1-4]. Já a polarização de concentração decorre do acúmulo de material na interface alimentação/membrana. Isso gera uma difusão dos solutos retidos em sentido oposto ao permeado, dificultando a permeação [1-4]. Em princípio, a polarização de concentração é um fenômeno reversível, mas ela contribui de forma significativa para o estabelecimento da incrustação, e, considerando a variedade de tipos de solutos, os fenômenos associados costumam ser muito complexos [11-14].

O objetivo deste trabalho foi preparar e caracterizar uma membrana polimérica de PEI pelo método da inversão de fases, usando NMP e água como solvente e não solvente, respectivamente. Foi escolhida como solução modelo uma emulsão óleo em água, simulando as características da água produzida [8]. Foram comparados sistemas com filtração frontal e tangencial, a fim de quantificar a queda no fluxo permeado e o coeficiente de rejeição. Com os fluxos encontrados, foram analisados os mecanismos de entupimento dos poros, através do modelo de Hermia [10]. O conhecimento do mecanismo que promove a queda do fluxo é de vital importância para programar a periodicidade e o tipo de limpeza a ser empregada no processo, visando estender a vida útil das membranas e aumentar sua produtividade.

\section{MATERIAIS E MÉTODOS}

\subsection{Preparo da membrana por inversão de fases}

Foram utilizados PEI, da Sigma Aldrich, e NMP, da Neon. A solução polimérica foi preparada com concentração 8\% em massa do polímero, por meio de agitação magnética durante 96 horas, até dissolução completa. Cerca de $3 \mathrm{~g}$ da solução foram transferidas para uma placa de vidro plana de $(20 \times 30) \mathrm{cm}^{2}$ com auxílio de um bastão de vidro. No caso da membrana utilizada em filtração tangencial, a quantidade de solução colocada na placa foi de, aproximadamente, $6 \mathrm{~g}$, ou seja, o dobro da quantidade utilizada para o preparo da membrana usada em filtração frontal. Logo em seguida, a placa foi mergulhada em banho com água (não solvente), para promover a separação de fases e originar a membrana. Após lavagem em outros três banhos de água, o filme polimérico foi seco por meio de trocas sucessivas de solventes, por cerca de 2 horas cada, a saber: 
etanol/água (50\% em massa), isopropanol e hexano, até completa secagem a $25^{\circ} \mathrm{C}$, em capela com exaustão ligada.

\subsection{Caracterização morfológica}

As membranas foram analisadas por microscopia eletrônica de varredura (JEOL JSM - 6360LV) para avaliar a estrutura morfológica da seção transversal. Utilizou-se nitrogênio líquido para promover a fratura da membrana, com auxílio de um bisturi. As amostras foram fixadas em suporte específico e metalizadas com uma fina camada de ouro (2-3 nm) por plasma (Baltec, MD20).

\subsection{Caracterização das propriedades de transporte}

As propriedades de transporte das membranas preparadas foram avaliadas pela microfiltração de uma emulsão óleo em água sintética simulando água produzida. Esta emulsão foi preparada misturando-se $30 \mathrm{~mL}$ de óleo de soja comercial (Liza), 3,6 g de dodecil sulfato de sódio (SDS, 90\% de pureza, Synth) com água destilada até o volume total de $1 \mathrm{~L}$. A emulsão foi homogeneizada usando um agitador mecânico, com rotação de $1000 \mathrm{rpm}$, durante 5 minutos.

O sistema experimental de filtração é apresentado na Figura 1. No caso da filtração tangencial, a alimentação foi bombeada para o módulo de membrana após passagem por um rotâmetro. Para a filtração frontal, a alimentação foi feita por meio de um funil de decantação, visando manter o nível de líquido constante durante o experimento. Os permeados foram recolhidos em proveta para determinação do fluxo e do coeficiente de rejeição.

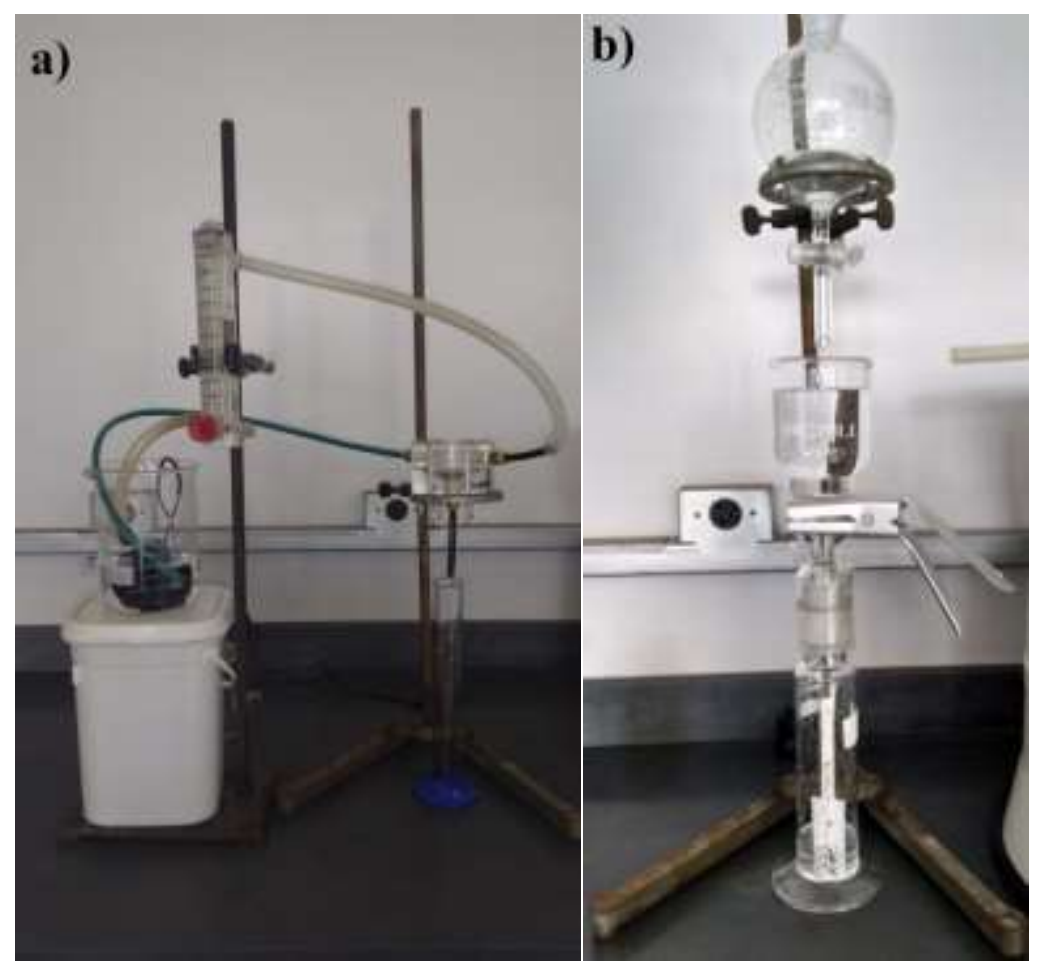

Figura 1: Esquema da montagem experimental utilizada nos ensaios de microfiltração: a) filtração com escoamento tangencial, b) filtração com escoamento frontal.

Inicialmente, o fluxo de água deionizada foi determinado. Em seguida, utilizou-se a emulsão o/a para determinar a queda do fluxo com o tempo e o coeficiente de rejeição da membrana. Após a permeação da suspensão, utilizou-se água destilada novamente para caracterizar a redução do fluxo após limpeza física da membrana com água. O volume utilizado nos testes foi de $250 \mathrm{~mL}$ de alimentação. Nos ensaios em que a alimentação escoou tangencialmente à membrana, utilizou-se vazão de circulação de $1000 \mathrm{~mL} \mathrm{~min}^{-1}$, área de membrana de 13,2 $\mathrm{cm}^{2}$ e pressão transmembranar de 9,7 x $10^{-4}$ atm. Para o caso da filtração frontal, utilizouse área de permeação de $9,6 \mathrm{~cm}^{2}$ e pressão transmembranar de $4,8 \times 10^{-3} \mathrm{~atm}$.

O fluxo do permeado (J) foi calculado a partir da equação (1): 


$$
J=\frac{1}{A} \frac{d V_{p}}{d t}
$$

onde, A é a área da membrana e $\mathrm{dV}_{\mathrm{p}} / \mathrm{dt}$ é a vazão de permeado. Os fluxos de água, de suspensão inicial, de suspensão final e de água após limpeza foram denominados $\mathrm{J}_{\mathrm{a}}, \mathrm{J}_{\mathrm{si}}, \mathrm{J}_{\mathrm{sf}}$ e $\mathrm{J}_{\mathrm{af}}$, respectivamente. Considerando as diferentes condições experimentais, os fluxos foram comparados em relação ao de água, $\mathrm{J}_{\mathrm{a}}$. Para a análise de declínio do fluxo total $\left(\mathrm{DF}_{\mathrm{t}}\right)$, reversível $\left(\mathrm{DF}_{\mathrm{r}}\right)$ e irreversível $\left(\mathrm{DF}_{\mathrm{i}}\right)$, foram utilizadas as equações de (2) a (4):

$$
\begin{aligned}
& D F_{t}=\left(\frac{J_{a}-J_{s f}}{J_{a}}\right) 100 \\
& D F_{r}=\left(\frac{J_{a f}-J_{s f}}{J_{a}}\right) 100 \\
& D F_{i}=\left(\frac{J_{a}-J_{a f}}{J_{a}}\right) 100
\end{aligned}
$$

A partir das medidas de turbidez (2130 B- Hach DR 2800) [15], foi também avaliada a rejeição da membrana, conforme equação (5):

$$
R(\%)=\frac{c_{f}-c_{p}}{C_{f}} 100
$$

onde $\mathrm{C}_{\mathrm{f}}$ e $\mathrm{C}_{\mathrm{p}}$ são a turbidez na alimentação e no permeado, respectivamente.

\subsection{Análise do mecanismo de incrustação na membrana}

Os resultados obtidos para o comportamento do fluxo com o tempo foram ajustados ao modelo de Hermia [10], conforme Tabela 1, visando identificar, a partir do coeficiente de correlação, $\mathrm{R}^{2}$, qual mecanismo explica melhor o tipo de entupimento dos poros das membranas em função do tipo de módulo operado. Os ajustes dos modelos foram realizados utilizando o MS Excel.

Tabela 1: Modelos de entupimento de acordo com o modelo de Hermia

\begin{tabular}{l|l}
\hline TIPO DE MECANISMO & EQUAÇÃO \\
\hline Bloqueio completo & $\ln \left(\mathrm{J}^{-1}\right)=\ln \left(\mathrm{J}_{0}^{-1}\right)+\mathrm{kt}$ \\
\hline Bloqueio padrão & $\mathrm{J}^{-1 / 2}=\mathrm{J}_{0}^{-1 / 2}+\mathrm{kt}$ \\
\hline Bloqueio intermediário & $\mathrm{J}^{-1}=\mathrm{J}_{0}^{-1}+\mathrm{kt}$ \\
\hline Formação de torta & $\mathrm{J}^{-2}=\mathrm{J}_{0}^{-2}+\mathrm{kt}$ \\
\hline
\end{tabular}

Nos modelos acima, $\mathbf{J}$ e $\mathbf{J}_{0}$ são os fluxos em um tempo qualquer e no tempo inicial, k é o coeficiente de transferência de massa e t é o tempo do experimento.

\section{RESULTADOS E DISCUSSÃO}

\subsection{Caracterização morfológica}

A microscopia eletrônica de varredura foi utilizada para analisar a morfologia transversal da membrana preparada. As micrografias são apresentadas na Figura 2. Observou-se uma estrutura assimétrica em ambas as membranas, mais densificada junto à superfície e com poros no formato de dedos, conforme esperado [6,7]. A extração lenta do solvente em virtude da grande viscosidade da solução favoreceu alta porosidade e maior tamanho de poros [6]. 


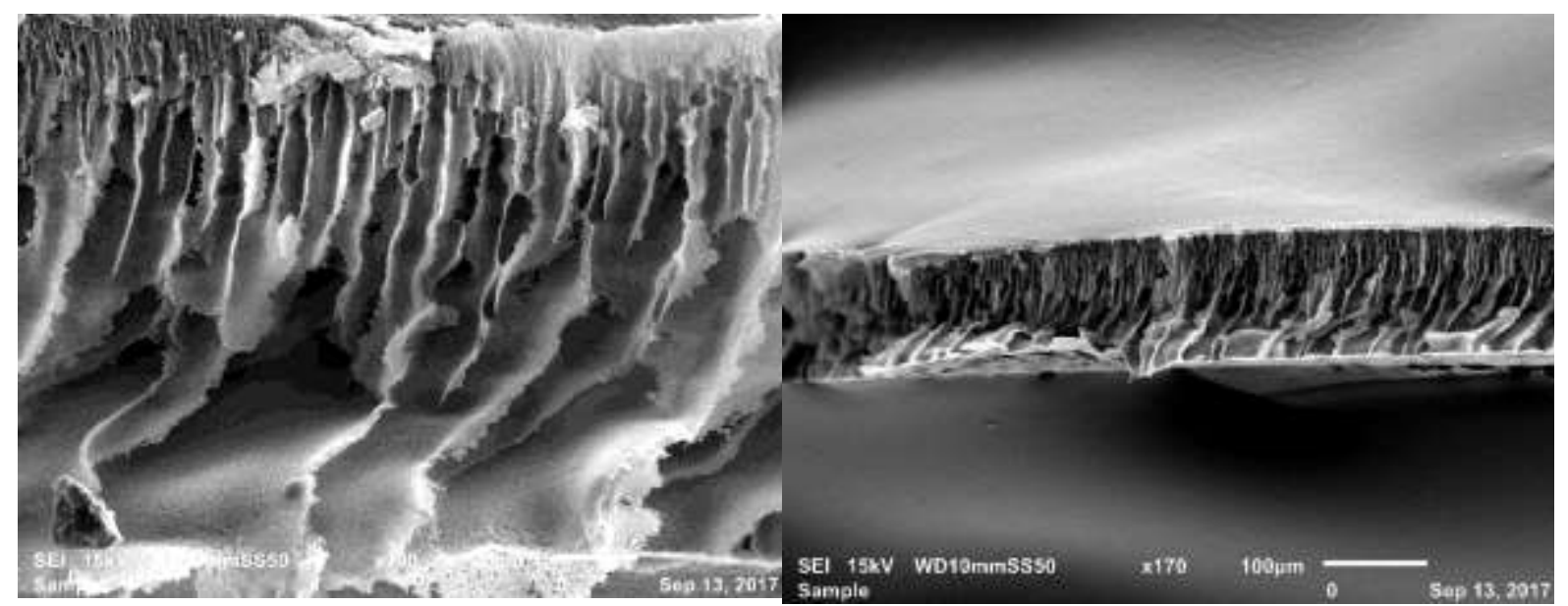

a

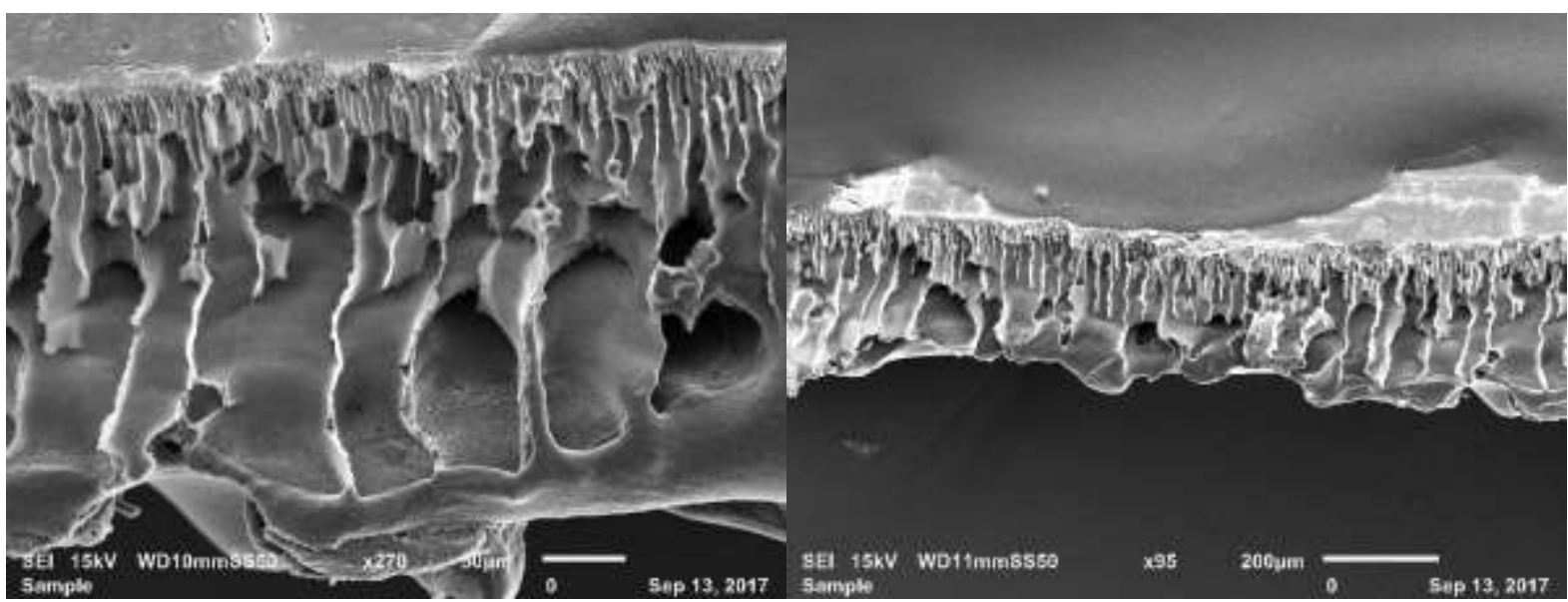

b

Figura 2: Micrografia das membranas de polieterimida preparadas pelo método de inversão de fases: a) escoamento frontal, b) escoamento tangencial

As membranas usadas em filtração tangencial apresentaram espessura média de $(260 \pm 23) \mu \mathrm{m}$, com tamanho médio de poros de $(10,4 \pm 0,6) \mu \mathrm{m}$. Já a membrana utilizada nos testes de filtração frontal apresentou espessura média de $(124 \pm 4) \mu \mathrm{m}$, com poros de diâmetro igual a $(1,2 \pm 0,3) \mu \mathrm{m}$. As médias e desvios apresentados correspondem às medidas em triplicata das imagens apresentadas na Figura 2. Estes resultados ilustram o potencial da técnica de inversão de fases para a produção de membranas com diferentes porosidades e tamanho de poros, tendo em vista o controle das condições cinéticas de precipitação [6,7].

\subsection{Caracterização das propriedades de transporte}

Os resultados obtidos para o fluxo relativo, o declínio do fluxo e suas frações, bem como a rejeição são apresentados na Tabela 2.

Tabela 2: Resultados de declínio do fluxo em função do módulo de permeação empregado.

\begin{tabular}{l|l|l|l|l|l|l|l}
\hline \multirow{2}{*}{ FILTRAÇÃO } & \multicolumn{3}{l|}{ FLUXO $\left(\mathbf{L ~ M}^{-2} \mathbf{H}^{-1}\right)$} & \multicolumn{3}{l|}{ DECLíNIO DO FLUXO (\%) } & COEFICIENTE DE \\
\cline { 2 - 8 } & $\mathrm{J}_{\mathrm{a}}$ & $\mathrm{J}_{\mathrm{si}}-\mathrm{J}_{\mathrm{sf}}$ & $\mathrm{J}_{\mathrm{af}}$ & $\mathrm{DF}_{\mathrm{t}}$ & $\mathrm{DF}_{\mathrm{r}}$ & $\mathrm{DF}_{\mathrm{i}}$ & REJEIÇÃO (\%) \\
\hline Frontal & 205,2 & $33,7-5,5$ & 70,6 & 97,3 & 65,6 & 31,7 & 95,5 \\
\hline Tangencial & 32,5 & $4,21-3,4$ & 16,8 & 89,5 & 48,3 & 41,2 & 88,9 \\
\hline
\end{tabular}


Os valores de fluxo $\left(\mathrm{J}_{\mathrm{a}}, \mathrm{J}_{\mathrm{si}}, \mathrm{J}_{\mathrm{sf}}\right.$ e $\left.\mathrm{J}_{\mathrm{af}}\right)$ foram muito discrepantes para o sistema de filtração frontal e tangencial devido tanto às diferenças estruturais das membranas preparadas quanto das condições operacionais empregadas nos testes. Destaca-se que o uso de membranas mais espessas nos testes de filtração tangencial foi necessário devido às tensões de cisalhamento provocadas pelo escoamento da alimentação, que promoveram a ruptura de membranas mais finas. O declínio de fluxo e de suas parcelas foi, então, avaliado em relação ao fluxo inicial de água, $\mathrm{J}_{\mathrm{a}}$, em cada condição.

Os resultados indicaram que a passagem da emulsão o/a sintética pela membrana promoveu uma redução de até 3\% do fluxo, comparado com água pura. O maior declínio do fluxo foi observado para o sistema em que a filtração ocorreu no modo frontal, conforme esperado, já que o material retido impede a passagem de mais solução pela membrana, reduzindo a área efetiva para permeação [13]. Para o escoamento tangencial, a redução do fluxo permeado não só foi menor, quanto maior foi a quantidade do fluxo recuperado após limpeza física da membrana (51,7\% em comparação com 34,4 \% na filtração frontal). Os resultados sugerem, portanto, que o modo tangencial promove uma melhor hidrodinâmica do sistema ao longo do tempo de operação, que foi em torno de 50 minutos de testes [14].

Tanto no modo de operação frontal quanto no tangencial, observou-se que a maior contribuição para a redução do fluxo foi reversível, indicando que a polarização de concentração foi o principal fator que influenciou o sistema. Isso indica que a utilização da membrana para o tratamento de água de produção é bastante promissora, já que é possível reverter os efeitos causadores da queda de fluxo com o tempo [12].

Quanto aos coeficientes de rejeição, observou-se que a maior rejeição foi obtida para o sistema operado em modo frontal, conforme esperado, já que o acúmulo de material na superfície da membrana é maior, melhorando a qualidade do permeado obtido.

\subsection{Modelagem dos mecanismos de bloqueio de poros}

Os parâmetros estimados pelos modelos de Hermia para análise do comportamento do fluxo com o tempo são apresentados na Tabela 3. O modelo de Hermia consiste em quatro tipos básico de fouling: bloqueio completo, intermediário e padrão dos poros e a formação de torta [10]. Os dois primeiros acontecem quando as gotas da emulsão são maiores que o tamanho dos poros e vão se acumulando umas nas outras formando uma monocamada ou selando a alguns poros, respectivamente. No bloqueio padrão, ocorre a adsorção do soluto nos poros, ou seja, as gotas da emulsão têm um tamanho muito menor que o poro da membrana, diminuindo seu diâmetro. A formação da torta ocorre pela deposição de gotas da emulsão na superfície da membrana, formando uma camada que dificulta ainda mais a filtração [16-18].

Tabela 3: Ajuste paramétrico e qualidade dos modelos de Hermia aplicados aos dados experimentais.

\begin{tabular}{l|l|l|l|l|l|l}
\hline \multirow{2}{*}{ MODELO } & \multicolumn{5}{l}{ FILTRAÇÃO FRONTAL } & \multicolumn{3}{l}{ FILTRAÇÃO TANGENCIAL } \\
\cline { 2 - 8 } & $\mathrm{J}_{0}\left(\mathrm{Lm}^{-2} \mathrm{~h}^{-1}\right)$ & $\mathrm{k}$ & $\mathrm{R}^{2}$ & $\mathrm{~J}_{0}\left(\mathrm{Lm}^{-2} \mathrm{~h}^{-1}\right)$ & $\mathrm{k}$ & $\mathrm{R}^{2}$ \\
\hline Completo & 26,17 & 0,3073 & 0,8926 & 4,14 & 0,0296 & 0,8150 \\
\hline Padrão & 28,02 & 0,0446 & 0,9581 & 4,15 & 0,0076 & 0,8252 \\
\hline Intermediário & 32,36 & 0,0273 & 0,9931 & 4,15 & 0,0078 & 0,8351 \\
\hline Formação de torta & -- & 0,0058 & 0,9824 & 4,16 & 0,0041 & 0,8540 \\
\hline
\end{tabular}

Para o caso da filtração frontal, observou-se que o modelo que melhor se ajustou aos dados experimentais foi o de bloqueio intermediário dos poros, segundo o qual há obstrução destes por espécies ou gotas de tamanhos similares a eles. É menos restritivo que o modelo de bloqueio completo, uma vez que a sobreposição das gotas é considerada e, portanto, nem toda gota que se aproximar da membrana poderá bloquear um poro, podendo depositar-se sobre os demais [18]. Cumpre destacar que, muito embora o ajuste matemático do modelo de formação de torta tenha sido também elevado, com $\mathrm{R}^{2}$ muito semelhante ao bloqueio intermediário, o parâmetro obtido para o fluxo inicial não possui significado físico, já que retornou um número complexo. Resultados semelhantes foram observados na literatura para a ultrafiltração frontal de efluente com elevada turbidez e salinidade [19].

Os mecanismos de declínio de fluxo do modelo de Hermia para sistema com escoamento tangencial preveem a redução contínua do fluxo permeado até valores nulos, o que é alcançado na prática para tempos muito longos de operação. Isso que garante a validade do modelo para este modo de operação [17,18]. A partir da análise da Tabela 3, observou-se que a qualidade do ajuste foi menor para este modo de operação, conforme observado pelos valores de $\mathrm{R}^{2}$, sempre menores do que aqueles observados no modo frontal. Isso pode 
ser justificado pelo fato de o sistema ter alcançado um fluxo pseudoestacionário durante o experimento, sem alcançar valores nulos. Ainda assim, vale ressaltar que o principal mecanismo observado foi o de formação de torta, indicando que, provavelmente, as maiores gotas da alimentação foram empurradas para a interface com a membrana preferencialmente às demais. Embora a queda no fluxo seja significativa neste caso, vale destacar que nestas condições a retomada do fluxo pode ser facilmente alcançada a partir da retrolavagem periódica da membrana [12]. É possível também que mais de um mecanismo esteja atuando e isso justifique a pior qualidade do ajuste neste modo de operação.

\section{CONCLUSÕES}

Membranas anisotrópicas de microfiltração compostas por PEI/NMP/água foram preparadas com propriedades morfológicas diferentes a partir da mudança na cinética de precipitação. Os resultados de permeação revelaram que grande parcela do fluxo foi recuperado a partir de limpeza física das membranas, indicando o potencial de aplicação do mesmo, desde que haja retrolavagem periódica durante a operação. Os mecanismos de declínio do fluxo que melhor se ajustaram aos dados de permeação foram a formação de torta e o bloqueio intermediário dos poros, o que confirma o potencial do sistema para operação industrial, desde que seja realizada limpeza periódica das membranas.

\section{AGRADECIMENTOS}

As autoras agradecem à Capes, Fapemig e CNPq pelo auxílio para o desenvolvimento desta pesquisa.

\section{BIBLIOGRAFIA}

[1] BAKER, R. W., Membrane Technology and Applications, 3 ed., New York, Wiley, 2012.

[2] HABERT, A. C., BORGES, C. P., NÓBREGA, R., Processos de Separação por Membranas, Rio de Janeiro, e-papers, 2006.

[3] MULDER, M., Basic Principles of Membrane Technology, London, Kluwer, 1996.

[4] NOBLE, R. D., STERN, S. A. Membrane separation technology: Principle and Applications, 3 ed., Amsterdan, Elsevier, 2003.

[5] ZHANG, Y., ZHONG, M., LUO, B., LI, J., YUAN, Q., YANG, X. J., "The performance of integrally skinned polyetherimide asymmetric nanofiltration membranes with organic solvents", Journal of Membrane Science, v. 544, pp. 119-125, Dec. 2017.

[6] BAKERI, G., MATSUURA, T., ISMAIL, A. F., "The effect of phase inversion promoters on the structure and performance of polyetherimide hollow fiber membrane using in gas-liquid contacting process", Journal of Membrane Science, v. 383, n. 1-2, pp. 159-169, Nov. 2011.

[7] REN, J., ZHOU, J., DENG, M., "Morphology transition of asymmetric polyetherimide flat sheet membranes with different thickness by wet phase-inversion process", Separation and Purification Technology,v. 74, n. 1, pp. 119-129, Jul. 2010.

[8] CARMO, S., NETO, T. L. A., OLIVEIRA, G. N., CASTRO, V. D., FIGUEIREDO, K. C. S., "Tratamento de água produzida de petróleo utilizando microfiltração", E-xacta, v. 10, n. 2, pp. 95-106, Dec 2017.

[9] DICKOUT, J. M., MORENO, J., BIESHEUVEL, P. M., BOELS, L., LAMMERTINK, R. G. H., DE VOS, W. M., "Produced water treatment by membranes: A review from a colloidal perspective", Journal of Colloid and Interface Science, v. 487, pp. 523-534, Feb. 2017.

[10] HERMIA, J., "Constant pressure blocking filtration laws-application to power-law non-newtonian fluids", Trans. Inst. Chem. Eng., v. 60, pp. 183-187, 1982.

[11] HUANG, S., RAS, R. H. A., TIAN, X., "Antifouling membranes for oily wastewater treatment: interplay between wetting and membrane fouling", Current Opinion in Colloid \& Interface Science (2018), doi: 10.1016/j.cocis.2018.02.002.

[12] BELFORT, G., DAVIS, R. H., ZYDNEY, A. L., "The behavior of suspensions and macromolecular solutions in crossflow microfiltration", Journal of Membrane Science, v. 96, n. 1-2, pp. 1-58, Nov. 1994.

[13] VElasCo, C., CAlVO, J. I., PALACIO, L., CARMONA, J., PRÁDANOS, P., HERNNÁNDES, A., "Flux kinetics, limit and critical fluxes for low pressure dead-end microfiltration. The case of BSA filtration through a positively charged membrane", Chemical Engineering Science, v. 129, pp. 58-68, Jun 2015.

[14] WANG, Z., CHU, J., WU, W., YAO, J., "Study of unsteady state flux prediction ion cross-flow microfiltration”, Desalination, v. 238, n. 1-3, pp. 290-301, Mar. 2009. 
[15] APHA, AWWA, WEF, Standard methods for the examination of water and Wastewater, 22 ed, Washington, American Public Health Association, 2005.

[16] CORBATON-BAGUENA, M. J., VELA, M. C. V., ZAFRILlA, J. M. G., BLANCO, S. A., GARCIA, J. L., MARTINEZ, D. C., "Comparison between artificial neural networks and Hermia's models to assess ultrafiltration performance", Separation and Purification Technology, v. 170, pp. 434-444, Oct 2016.

[17] KAYA, Y., GONDER, Z. B., VERGILI, I., BARLAS, H., "The effect of transmembrane pressure and $\mathrm{pH}$ on treatment of paper machine process waters by using a two-step nanofiltration process: Flux decline analysis", Desalination, v. 250, n. 1, pp. 150-157, Jan 2010.

[18] VELA, M. C. V., BLANCO, S. A., GARCIA, J. L., RODRIGUES, E. B., “Analysis of membrane pore blocking models applied to the ultrafiltration of PEG", Separation and Purification Technology, v. 62, n. 3, pp. 489-498, Sep. 2008.

[19] FRATIlA-APACHITEI, L. E., KENNEDY, M. D., LINTON, J. D., BLUME, I., SCHIPPERS, J. C., "Influence of membrane morphology on the flux decline during dead-end ultrafiltration of refinery and petrochemical wastewater", Journal of Membrane Science, v. 182, n. 1-2, pp. 151-159, Feb. 2001.

\section{ORCID}

Evelin Campos Verdolin Brandão https://orcid.org/0000-0002-3284-1547

Kátia Cecília de Souza Figueiredo

https://orcid.org/0000-0001-7207-1535 